\title{
Raman Spectroscopy of Shear Modes in a Few-Layer $\mathrm{MoS}_{2}$
}

\author{
K. Golasa ${ }^{a}, *$, M. GrzeszczyK ${ }^{a}$, M. Zinkiewicz ${ }^{a}$, K. Nogajewski ${ }^{b}$, M. Potemski $^{b}$, \\ A. WYSmoŁeK ${ }^{a}$ AND A. BABIŃSKI ${ }^{a}$ \\ ${ }^{a}$ Faculty of Physics, University of Warsaw, L. Pasteura 5, 02-093 Warszawa, Poland \\ ${ }^{b}$ Laboratoire National des Champs Magnetiques Intenses, CNRS-UJF-UPS-INSA, \\ 25, av. des Martyrs, 38042 Grenoble, France
}

\begin{abstract}
We study low frequency vibrational modes in atomically thin molybdenum disulfide $\left(\mathrm{MoS}_{2}\right)$ by means of the Raman scattering spectroscopy. A shear mode related to rigid interlayer vibrations is identified. Its energy evolution with the increasing number of layers is well described using a linear chain model with only nearest neighbor interactions. The resulting force constant $\left(K_{x}=2.7 \times 10^{19} \mathrm{~N} / \mathrm{m}^{3}\right)$ corresponds well to the previously published data.
\end{abstract}

DOI: 10.12693/APhysPolA.129.A-132

PACS: 78.20.-e, 87.64.Je, 63.20.Kr

\section{Introduction}

Atomically thin layered transition metal dichalcogenide compounds (TMD) $\mathrm{MX}_{2}$, where $\mathrm{M}=$ transition metal, $\mathrm{X}=\mathrm{S}$, Se, Te, are of considerable interest due to their unique physical and optical properties [1]. Strong ion-covalent bonds within planes of hexagonally arranged $\mathrm{X}$ and $\mathrm{M}$ atoms and weak out-of-plane van der Waals interactions between the planes make their properties very strongly dependent on the number of layers which form the structure. In particular the transition from the indirect bandgap in bulk TMDs to the direct bandgap in their two-dimensional form [2] can be exploited in several optoelectronic applications [3]. Possible applications and interesting physics justify investigation of basic properties of a few layer TMDs and in particular studies of their lattice dynamics. Phonons are important for several physical processes as e.g. carrier scattering [4], heat propagation [5], and mechanical strength of the crystal [6]. Mechanical properties of TMDs are determined by force constants resulting from interlayer interactions. Following several recent contributions [7-10] we study the Raman scattering spectroscopy in a low-energy range in which the rigid layer modes can be observed. We identify the shear mode and we follow its dependence on the number of layers. We show that the energy evolution of the mode with the increasing number of layers can be well described using a linear chain model with only nearest neighbor interactions. The in-plane shear force constant is determined.

\section{Experimental}

The sample was prepared by thinning down $\mathrm{MoS}_{2}$ crystal by exfoliation with a high-quality backgrinding tape. An all-dry, non-deterministic, polydimethylsiloxan-based

*corresponding author; e-mail: Katarzyna.Golasa@fuw.edu.pl stamping technique was employed to transfer the sample onto a $\mathrm{Si} / 90 \mathrm{~nm} \mathrm{SiO}_{2}$ substrate. Optical spectroscopic measurements were performed in a backscattering geometry using $\lambda=532 \mathrm{~nm}(2.33 \mathrm{eV})$. The $\mathrm{He}-\mathrm{Ne}$ laser with $\lambda=632.8 \mathrm{~nm}(1.96 \mathrm{eV})$ excitation was also used for comparative studies. At room temperature the laser light power on the sample was equal to $\approx 190 \mu \mathrm{W}$. An Olympus MPlan N microscope 100/0.90 objective was used to both excite the sample and to collect the emitted light. The collected spectra were dispersed by a HoribaYobin T64000 Series II $\mu$-Raman system, equipped with a multichannel high-resolution Si-charge coupled device. The spectrometer was set to a tri-grid measuring mode (with a grating of $1800 \mathrm{l} / \mathrm{mm}$ ).

\section{Results and discussion}

In our study we focus on the low-energy features observed at the energies below $60 \mathrm{~cm}^{-1}$. One expects two families of interlayer modes in the low-energy range, which correspond to the rigid vibrations of $\mathrm{MoS}_{2}$ layers. These are shear modes related to rigid layer displacements perpendicular to the $c$ axis and breathing modes which correspond to rigid layer displacements along the $c$ axis [10-12]. The modes evolve into the Raman active $E_{2 \mathrm{~g}}^{2}$ and optically inactive $B_{2 \mathrm{~g}}^{2}$ in bulk, respectively. The evolution of the low-energy Raman spectrum with changing number of layers $(N)$ is presented in Fig. 1. It can be seen that the spectrum critically depends on the number of layers.

There are no interlayer modes in monolayer $\mathrm{MoS}_{2}$, which explains the lack of any features in the corresponding spectrum. Starting from bilayer (2ML) there is just one clearly observed peak in the spectrum and its frequency increases with $N$. Eventually, in bulk the peak corresponds to $E_{2 \mathrm{~g}}^{2}$ Raman active mode. This suggests that the peaks represent the interlayer shear modes. Beyond the model, another peak is present (denoted in Fig. 1 with a star) in the Raman spectrum from hexalayer $(N=6)$, its possible origin is discussed later in the text. 


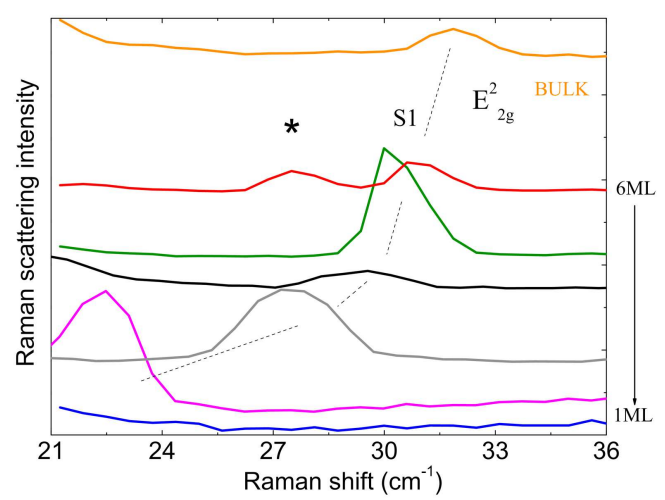

Fig. 1. Low-frequency Raman spectra as a function of the $\mathrm{MoS}_{2}$ number of layers. The spectra are off-set for clarity. Dashed lines, which follow the S1 peak are the guides to the eye.

In our analysis of the evolution of the Raman peaks due to the shear modes we employ the linear chain model of the crystal lattice vibrations. Whole layers of atoms are represented in the model by a single point of mass which represents the surface mass density of the atoms, composing the layer. Only the interaction between the adjacent layers is retained which is described by a single force constant $K_{i} . K_{x}$ and $K_{z}$ correspond to the shearing and compression strength, respectively. The approach has recently proved to describe reasonably the rigid modes in $\mathrm{MoS}_{2}$ and $\mathrm{WeSe}_{2}[10,12]$.

The evolution of the mode energies $\left(\right.$ in $\mathrm{cm}^{-1}$ ) as a function of $N$-layers can be represented within the model by

$$
\begin{gathered}
\omega_{i, \alpha}=\sqrt{\frac{K_{i}}{2 \mu \pi^{2} c^{2}}\left[1-\cos \left(\frac{(\alpha-1) \pi}{N}\right)\right]}= \\
\omega_{i} \sqrt{1-\cos \left(\frac{(\alpha-1) \pi}{N}\right)}
\end{gathered}
$$

with $\alpha=2,3, \ldots, N$. The reduced mass $\mu=3 \times$ $10^{-6} \mathrm{~kg} / \mathrm{m}^{2}$ is the rigid layer mass per unit area $(\mu=$ $2 m_{\mathrm{S}}+m_{\mathrm{Mo}}$, where $m_{\mathrm{S}}=0.6 \times 10^{-6} \mathrm{~kg} / \mathrm{m}^{2}$ for $\mathrm{S}$ atoms and $m_{\mathrm{Mo}}=1.8 \times 10^{-6} \mathrm{~kg} / \mathrm{m}^{2}$ for Mo, and $c$ is the speed of light in $\mathrm{cm}^{-1}$.

There are two sets of subbranches expected for shear (breathing) modes. The Raman active shear (breathing) modes belong to the higher-energy (lower-energy) branches of the corresponding set.

We start our analysis with the mode $E_{2 \mathrm{~g}}^{2}$ which can be observed in bulk at $31.9 \mathrm{~cm}^{-1}$. Assuming that the force constant $K_{x}$ does not change significantly as $N$ changes from 2 to $N \longrightarrow \infty$ [13] one can predict the value of $\omega_{x}=22.6 \mathrm{~cm}^{-1}$. The energy of the shear mode in bilayer predicted by the model corresponds to the energy of one of the modes observed in our experiment at $22.5 \mathrm{~cm}^{-1}$. Using the value one can model the evolution of the shear modes with $\alpha=N$ and $N-1$, which are denoted in Fig. 2 with continuous and dashed line, respectively. It can be seen that the subbranch modelled with $\alpha=N$ reproduces well the observed evolution of the main modes, which stiffen with increasing number of layers. The $\omega_{x, N}$ subbranch corresponds to previously observed S1 shear mode in $\mathrm{MoS}_{2}$ and $\mathrm{WSe}_{2}$ [10] which is also referred to as $C_{2}$ [14]. Using the value of $\omega_{x}=22.6 \mathrm{~cm}^{-1}$ one can also find the in-plane (shear) force constant $K_{x}=2.7 \times 10^{19} \mathrm{~N} / \mathrm{m}^{3}$ which perfectly matches the previously reported value [10] and which is smaller than the value for $\mathrm{WSe}_{2}\left(3.1 \times 10^{19} \mathrm{~N} / \mathrm{m}^{3}\right)[13]$.

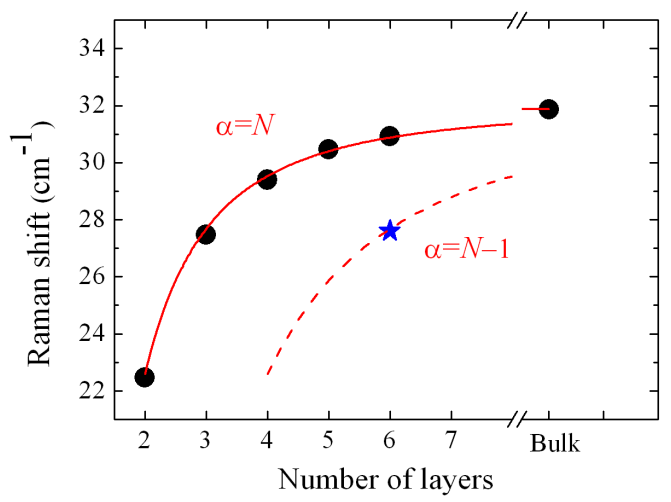

Fig. 2. The frequency evolution of shear modes as a function of $\mathrm{MoS}_{2}$ number of layers.

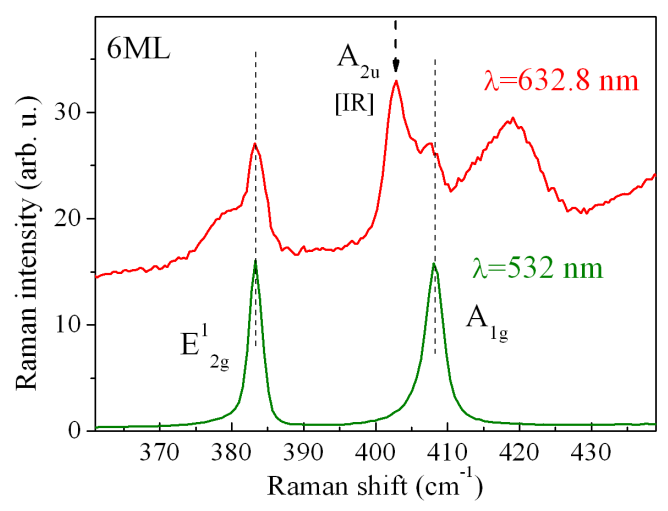

Fig. 3. Resonant vs. non-resonant Raman scattering in $6 \mathrm{ML} \mathrm{MoS}_{2}$.

The most intriguing property of the observed Raman spectrum is the peak at $27.5 \mathrm{~cm}^{-1}$ observed in $6 \mathrm{ML}$ sample (see Fig. 2). As it can be seen in Fig. 2 the mode coincides with the expected energy of a shear mode, which belongs to $\alpha=N-1$ subbranch, and which is not normally observed in the Raman spectrum. In our opinion the brightening of the mode must be due to the strain or defects present in $6 \mathrm{ML}$ flake. The same effect must be also responsible for the unexpected lineshape of the out-of-plane mode $\left(A_{1 \mathrm{~g}}\right)$ in the spectrum excited resonantly with $\lambda=632 \mathrm{~nm}$ (see Fig. 3 ). The additional line corresponds most likely to $A_{2 \mathrm{u}}$ IR-active mode in $6 \mathrm{ML} \mathrm{MoS}_{2}$ however the mechanism of its brightening is also not clear at the moment. 


\section{Conclusions}

We have studied low frequency vibrational modes in atomically thin $\mathrm{MoS}_{2}$ by means of the Raman scattering spectroscopy. A shear mode related to rigid interlayer vibrations has been identified. Its energy evolution with the increasing number of layers has been described using a linear chain model with only nearest neighbor interactions. The resulting force constant $\left(K_{x}=2.7 \times 10^{19} \mathrm{~N} / \mathrm{m}^{3}\right)$ has been found to correspond well to the previously published data.

\section{Acknowledgments}

This work has been supported by the National Science Centre under grants no. DEC 2013/11/N/ST3/04067 and DEC-2013/10/M/ST3/00791. Funding from European Graphene Flagship and European Research Council (ERC-2012-AdG-320590-MOMB) is also acknowledged. The support by The Board of the Mazovian Voivodeship within the framework of the project: "Development of science - the development of the region - supporting scholarships and support for Mazovia PhD" is also acknowledged.

\section{References}

[1] S.Z. Butler, S.M. Hollen, L. Cao, Y. Cui, J.A. Gupta, H.R. Gutirrez, T.F. Heinz, S.S. Hong, J. Huang, A.F. Ismach, E. Johnston-Halperin, M. Kuno, V.V. Plashnitsa, R.D. Robinson, R.S. Ruoff, S. Salahuddin, J. Shan, L. Shi, M.G. Spencer, M. Terrones, W. Windl, J.E. Goldberger, ACS Nano 7, 2898 (2013).

[2] A. Kuc, N. Zibouche, T. Heine, Phys. Rev. B Condens. Matter Mater. Phys. 83, 245213 (2011).
[3] D. Jariwala, V.K. Sangwan, L.J. Lauhon, T.J. Marks, M.C. Hersam, ACS Nano 8, 1102 (2014).

[4] K. Kaasbjerg, K.S. Thygesen, A.-P. Jauho, Phys. Rev. B Condens. Matter Mater. Phys. 87, 235312 (2013).

[5] X. Liu, G. Zhang, Q.-X. Pei, Y.-W. Zhang, Appl. Phys. Lett. 103, 133113 (2013).

[6] T. Li, Phys. Rev. B Condens. Matter Mater. Phys. 85, 235407 (2012).

[7] H. Zeng, B. Zhu, K. Liu, J. Fan, X. Cui, Q.M. Zhang, Phys. Rev. B 86, 241301(R) (2012).

[8] X. Zhang, W.P. Han, J.B. Wu, S. Milana, Y. Lu, Q.Q. Li, A.C. Ferrari, P.H. Tan, Phys. Rev. B 87, 115413 (2013).

[9] G. Plechinger, S. Heydrich, J. Eroms, D. Weiss, C. Schuller, T. Korn, Appl. Phys. Lett. 101, 101906 (2012).

[10] Y.Y. Zhao, X. Luo, H. Li, J. Zhang, P.T. Araujo, C.K. Gan, J. Wu, H. Zhang, S.Y. Quek, M.S. Dresselhaus, Q.H. Xiong, Nano Lett. 13, 1007 (2013).

[11] J.-U. Lee, J. Park, Y.-W. Son, H. Cheong, Nanoscale 7, 3229 (2015).

[12] X. Zhang, W.P. Han, J.B. Wu, S. Milana, Y. Lu, Q.Q. Li, A.C. Ferrari, P.H. Tan, Phys. Rev. B Condens. Matter Mater. Phys. 87, 115413 (2013).

[13] W. Zhao, Z. Ghorannevis, K.K. Amara, J.R. Pang, M. Toh, X. Zhang, C. Kloc, P.H. Tan, G. Eda, Nanoscale 5, 9677 (2013).

[14] X. Zhang, X.-F. Qiao, W. Shi, J.-B. Wu, D.-S. Jiang, P.-H. Tan, Chem. Soc. Rev. 44, 2757 (2015). 